\title{
Holomorphic one-forms, fibrations over the circle, and characteristic numbers of Kähler manifolds
}

\author{
BY D. KOTSCHICK \\ Mathematisches Institut, LMU München, \\ Theresienstr. 39, 80333 München, Germany. \\ e-mail:dieter@math.1mu.de
}

(Received 10 June 2019; revised 5 November 2020; accepted 26 October 2020)

\section{Abstract}

We prove that the only relation imposed on the Hodge and Chern numbers of a compact Kähler manifold by the existence of a nowhere zero holomorphic one-form is the vanishing of the Hirzebruch genus. We also treat the analogous problem for nowhere zero closed oneforms on smooth manifolds.

2010 Mathematics Subject Classification: 32Q15 (Primary); 32Q55, 55N22, 57R20 (Secondary)

\section{Introduction}

By the Poincaré-Hopf theorem, a closed smooth manifold admits a vector field without zeros if and only if its Euler characteristic vanishes. Dually, the vanishing of the Euler number characterises manifolds admitting a one-form without zeros. However, if one considers only closed one-forms, then there is an additional constraint. By Tischler's theorem [18] the existence of a closed one-form without zeros is equivalent to the existence of a smooth fibration over the circle. Such a fibration implies the vanishing of the signature. Conversely, it was shown by Neumann [15] that the vanishing of the signature is the only constraint imposed on the oriented bordism class of a manifold by the existence of a fibration over the circle. In Theorem 5 in Section 2 below we give a small improvement on this result which implies in particular that the vanishing of the Euler characteristic and of the signature are the only relations imposed on the Betti and Pontryagin numbers of manifolds by the existence of a fibration over the circle.

The main purpose of this paper is to prove Kähler analogs of the above results. It is a consequence of Bott's localisation formula [4] that on a compact complex manifold with a holomorphic vector field without zeros all Chern numbers vanish. This led Carrell [5] to ask what one can say about the Chern numbers of complex manifolds admitting non-vanishing holomorphic one-forms. He found that in dimensions one and two all Chern numbers vanish for such manifolds, and he gave an example showing that this is no longer true in higher dimensions. Here we completely answer Carrell's question, not only for Chern numbers, but also for the Hodge numbers of Kähler manifolds. 
THEOREM 1. For a compact Kähler manifold admitting a nowhere zero holomorphic one-form the Hirzebruch genus vanishes. In other words, the Euler characteristic of the bundle of holomorphic p-forms vanishes for all p. Conversely, there are no other $\mathbb{Q}$-linear relations imposed on the Hodge and Chern numbers by the existence of a nowhere zero holomorphic one-form.

This explains Carrell's results since in complex dimensions up to two the Chern numbers are determined by the Hirzebruch genus, whereas in dimensions at least three they are not. For example, in dimension three the vanishing of the Hirzebruch genus is equivalent to the vanishing of $c_{1} c_{2}$ and of $c_{3}$, but says nothing about $c_{1}^{3}$. The gap between the two grows very quickly, since in complex dimension $n$ the Hirzebruch genus gives only $[(n+2) / 2]$ combinations of Chern numbers that must vanish, whereas the total number of Chern numbers equals the number of partitions of $n$.

Since the appearance of Carrell's paper [5] more than 40 years ago, his question about the Chern numbers of manifolds with nowhere zero holomorphic one-forms seems to have received little attention, although many algebraic geometers have investigated the link between nowhere zero holomorphic one-forms and the Kodaira dimension suggested by Carrell's example; see for example [11, 19] and the references given there. That work culminated recently in the paper of Popa and Schnell [16], where it was shown that a smooth complex projective variety with a nowhere zero holomorphic one-form cannot be of general type. The present paper grew out of an (unsuccessful!) attempt to find a topological proof of the result of Popa and Schnell.

Our proof of Theorem 1 will show that the conclusion about the vanishing of the Hirzebruch genus is true for all compact Kähler manifolds that admit a smooth fibration over the circle, and that no other relations are imposed on the Hodge and Chern numbers by the existence of such a fibration. Thus the analytic or geometric assumption about the existence of a nowhere zero holomorphic one-form has exactly the same consequences as the differential topological assumption of the existence of a smooth fibration over the circle, compare Proposition 7 and Section 4.

\section{Smooth manifolds fibering over the circle}

In this section we consider closed oriented smooth manifolds $M$.

LEMmA 2. If $M$ admits a smooth fibration $f: M \longrightarrow S^{1}$, then both its Euler number $\chi(M)$ and its signature $\sigma(M)$ vanish.

Proof. Since $f$ is a fibration over the circle, we can pull it back under coverings of $S^{1}$ by itself to obtain coverings of $M$ of arbitrarily large degree, which also fiber over the circle with the same fiber $F$. Since $\chi$ and $\sigma$ are multiplicative in coverings, if one of them was non-zero on $M$, then it would be unbounded on these coverings. However, since all these coverings fiber over $S^{1}$ with the same fiber $F$, their Betti numbers, and therefore their Euler characteristics and their signatures, are bounded in terms of the Betti numbers of $F$. This contradiction shows that $\chi(M)=0=\sigma(M)$.

We now want to show that there are no other relations between the Betti and Pontryagin numbers of manifolds fibering over the circle. The Pontryagin numbers are determined by the bordism class, so we shall consider the oriented bordism ring $\Omega_{*}^{S O}$ and the signature 
homomorphism on it. To keep track of the Betti numbers we work with the Poincaré ring $\mathcal{P}_{*}$ introduced in [14]. This is the ring of Poincaré polynomials of formal $\mathbb{Z}$-linear combinations of closed oriented manifolds, graded by the dimension. We shall use the structure of this ring, which was determined in [14, theorem 5].

Theorem 3. ([14]) Let $W, X, Y$ and $Z$ have degrees 1, 2, 3 and 4 respectively. The Poincaré ring $\mathcal{P}_{*} \subset \mathbb{Z}[t, z]$ is isomorphic, as a graded ring, to the quotient of the polynomial ring $\mathbb{Z}[W, X, Y, Z]$ by the homogeneous ideal $\mathcal{I}$ generated by

$$
W X-2 Y, \quad X^{2}-4 Z, \quad X Y-2 W Z, \quad Y^{2}-W^{2} Z .
$$

Here $W, X, Y$ and $Z$ are elements with the following Poincaré polynomials:

$$
P(W)=(1+t) z, \quad P(X)=2 t z^{2}, \quad P(Y)=\left(t+t^{2}\right) z^{3}, \quad P(Z)=t^{2} z^{4} .
$$

Clearly $W=S^{1}$, and $X, Y, Z$ correspond to the Poincaré polynomials of the formal differences $S^{1} \times S^{1}-S^{2}, S^{1} \times S^{2}-S^{3}$ and $S^{2} \times S^{2}-\mathbb{C} P^{2}$.

The Euler characteristic defines a homomorphism on $\mathcal{P}_{*}$ given by evaluating Poincaré polynomials at $t=-1$. We have the following consequence of the above structure result.

PROPOSITION 4. The kernel of the Euler characteristic homomorphism $\chi: \mathcal{P}_{*} \longrightarrow \mathbb{Z}[z]$ is generated by manifolds that fiber over $S^{1}$ and are zero-bordant.

Proof. By Theorem 3, $\operatorname{ker}(\chi)$ is generated by $W$ and $Y$, with $W=S^{1}$. Thus we only have to show that $Y$ can be taken to fiber over $S^{1}$, and to bound. We may think of $Y$ as the formal difference $T-S^{1} \times S^{2}$, where $T$ is any closed oriented 3-manifold with $b_{1}(T)=2$. The conclusion follows since we may take for $T$ the total space of a circle bundle over $T^{2}$ with non-zero Euler class.

Finally we can state the main result of this section.

THEOREM 5. Let $\alpha \in \mathcal{P}_{n}$ with Euler characteristic $\chi(\alpha)=0$, and $\beta \in \Omega_{n}^{\text {SO }}$ with signature $\sigma(\beta)=0$. Then there is a formal $\mathbb{Z}$-linear combination $M$ of closed oriented smooth $n$ manifolds all of which fiber smoothly over $S^{1}$ such that the Poincaré polynomial $P(M)=\alpha$ and, simultaneously, the oriented bordism class $[M]=\beta$.

Proof. First of all, since $\sigma(\beta)=0$, the result of Neumann [15] shows that there exists a possibly disconnected - smooth closed oriented $n$-manifold $N$ such that $[N]=\beta$, and every connected component of $N$ admits a smooth fibration over $S^{1}$. The proof of this result gives no control over the Betti numbers of $N$, beyond the vanishing of the Euler characteristic. However, we can adjust the Betti numbers to be those of $\alpha$ as follows.

Let $P(N)$ be the Poincaré polynomial of $N$. Then $\alpha-P(N) \in \mathcal{P}_{n}$ is in the kernel of $\chi$. By Proposition 4 this kernel is generated by (Poincaré polynomials of) manifolds that fiber over $S^{1}$ and are zero-bordant. Thus, without changing the oriented bordism class, we add to $N$ some additional $\mathbb{Z}$-linear combinations of manifolds that fiber over $S^{1}$ and have Poincaré polynomial $\alpha-P(N)$. We finally obtain an $M$ that fibers over the circle and has Poincaré polynomial $\alpha$ and oriented bordism class $\beta$.

We have now proved the following: 
COROLLARY 6. If a closed oriented manifold $M$ fibers smoothly over $S^{1}$ then $\chi(M)=$ $0=\sigma(M)$. Conversely, any set of Betti and Pontryagin numbers satisfying these constraints is realised by a $\mathbb{Z}$-linear combination of closed oriented manifolds all of which fiber over $S^{1}$.

\section{Holomorphic one-forms without zeros on Kähler manifolds}

To discuss the characteristic numbers of Kähler manifolds with nowhere zero holomorphic one-forms we shall use the following.

PROPOSITION 7. Let $X$ be a compact Kähler manifold with a nowhere zero holomorphic one-form. Then $X$ admits a smooth fibration $f: X \longrightarrow T^{2}$. A fortiori $X$ fibers over the circle.

Proof. Let $\varphi \in H^{0}\left(X ; \Omega^{1}\right)$ be a holomorphic one-form without zeros. Since $X$ is Kähler, $\varphi$ and its complex conjugate $\bar{\varphi}$ are closed. Therefore $\psi_{1}=\varphi+\bar{\varphi}$ and $\psi_{2}=i(\varphi-\bar{\varphi})$ are closed real one-forms. For their wedge product we have

$$
\psi_{1} \wedge \psi_{2}=(\varphi+\bar{\varphi}) \wedge i(\varphi-\bar{\varphi})=-2 i \varphi \wedge \bar{\varphi} .
$$

The right-handside is everywhere non-zero because $\varphi$ is. Thus the $\psi_{i}$ are everywhere nonzero, and, furthermore, everywhere linearly independent. As in the proof of Tischler's theorem [18] we now use the density of rational cohomology classes in the first de Rham cohomology of $X$. This allows us to approximate the $\psi_{i}$ arbitrarily closely in the $C^{0}$ topology by closed one-forms $\widetilde{\psi}_{i}$ with rational periods. After multiplying by constants, we obtain two closed one-forms with integral periods. If the approximation is close enough, then the $\widetilde{\psi}_{i}$ are still everywhere linearly independent, meaning that the differential of the smooth map obtained by integration of $\left(\widetilde{\psi}_{1}, \widetilde{\psi}_{2}\right)$ with respect to an arbitrary basepoint has rank 2 , and so one obtains a smooth submersion onto $\mathbb{R}^{2} / \mathbb{Z}^{2}$. This completes the proof.

Remark 8. Versions of the proof of Proposition 7 have already appeared in the literature repeatedly. The first reference I am aware of is the paper of Crew and Fried [7], where the argument appears in the proof of their Theorem 1. The argument also reappears in more recent papers, for example in [17], always without reference to [7]. However, all these papers are really concerned with non-vanishing holomorphic vector fields, and the holomorphic one-form only appears as an auxiliary tool in proving something about vector fields. I have spelled out the argument to make it clear that it has nothing to do with vector fields, holomorphic or otherwise.

Remark 9. It is not possible in general to choose the fibration $f: X \longrightarrow T^{2}$ in Proposition 7 to be holomorphic. For example, let $X$ be an Abelian surface that is not isogenous to a product of elliptic curves. Then $X$ has nowhere zero holomorphic one-forms, and fibers smoothly over $T^{2}$, but does not fiber holomorphically over an elliptic curve; cf. [1, example 2.1].

We now want to determine the relations that the existence of a nowhere zero holomorphic one-form imposes on the Hodge and Chern numbers of a compact Kähler manifold. For this we use the Chern-Hodge ring introduced in [14, section 6]. It is defined by considering finite linear combinations of equidimensional compact Kähler manifolds with coefficients in $\mathbb{Z}$, and identifying two such linear combinations if they have the same dimensions and the same Hodge and Chern numbers. The set of equivalence classes is naturally a graded ring, graded by the dimension, with multiplication induced by the Cartesian product of Kähler 
manifolds. This is the Chern-Hodge $\operatorname{ring} \mathcal{C H}_{*}$, whose structure was completely determined in [14]. We only state a weak consequence of that structure result [14, theorem 12] that is sufficient for our purposes here.

THEOREM 10. ([14]) Let $\beta_{1}=\mathbb{C} P^{1}, \beta_{2}, \beta_{3}, \ldots$ be $\mathbb{Z}$-linear combinations of Kähler manifolds forming a basis sequence for the complex bordism ring $\Omega_{*}^{U}$. Then the Chern-Hodge ring $\mathcal{C} \mathcal{H}_{*}$ is a quotient of $\mathbb{Z}\left[E, \beta_{1}, \beta_{2}, \beta_{3}, \ldots\right]$, where $E$ is an elliptic curve.

For any compact Kähler manifold $X$ of complex dimension $n$ we denote by

$$
\chi_{p}(X)=\chi\left(X ; \Omega^{p}\right)=\sum_{q=0}^{n}(-1)^{q} h^{p, q}(X)
$$

the Euler characteristic of the sheaf cohomology of the bundle of holomorphic $p$-forms. By the Hirzebruch-Riemann-Roch theorem, this Euler characteristic can be expressed as a linear combination of Chern numbers, which we abbreviate as $\operatorname{Td}_{p}(X)$.

The Hirzebruch genus

$$
\chi_{y}(X)=\left(\sum_{p=0}^{n} \chi_{p}(X) y^{p}\right) \cdot z^{n}
$$

defines a ring homomorphism

$$
\chi_{y}: \mathcal{C H}_{*} \longrightarrow \mathbb{Z}[y, z]
$$

We now come to the main result of this paper.

THEOREM 11. The following three ideals in $\mathcal{C H}_{*} \otimes \mathbb{Q}$ coincide:

(i) the kernel of the Hirzebruch genus $\chi_{y}: \mathcal{C H}_{*} \otimes \mathbb{Q} \longrightarrow \mathbb{Q}[y, z]$;

(ii) the ideal $\mathcal{I}$ generated by compact Kähler manifolds fibering smoothly over the circle; and

(iii) the ideal $\mathcal{J}$ generated by compact Kähler manifolds admitting a nowhere zero holomorphic one-form.

It should be obvious that this implies Theorem 1 stated in the introduction. The first assertion in Theorem 1 is just the inclusion $\mathcal{J} \subset \operatorname{ker}\left(\chi_{y}\right)$, and the second assertion is that this inclusion is not strict.

Proof of Theorem 11. Note first of all that the conditions that every connected component fiber smoothly over $S^{1}$ or that every connected component admit a nowhere zero holomorphic one-form do indeed define ideals in the Chern-Hodge ring.

Proposition 7 shows that $\mathcal{J} \subset \mathcal{I}$. Next we claim that $\mathcal{I} \subset \operatorname{ker}\left(\chi_{y}\right)$. The argument for this is the same as the proof of Lemma 2. In more detail, let $X$ be a compact Kähler manifold fibering smoothly over $S^{1}$, and assume that some $\chi_{p}(X)=\operatorname{Td}_{p}(X)$ does not vanish. Then because the right-hand side is multiplicative in finite coverings, this quantity is unbounded on finite coverings of $X$ induced by finite coverings of $S^{1}$ over itself. However, the left-hand side is by definition a linear combination of Hodge numbers, and, since $X$ is Kähler, this 
is bounded in terms of Betti numbers. Now the Betti numbers of a sequence of coverings of $X$ induced by coverings of $S^{1}$ are bounded in terms of the Betti numbers of the fiber of $f: X \longrightarrow S^{1}$. This shows that $\chi_{p}(X)=\operatorname{Td}_{p}(X)$ must vanish after all.

To complete the proof of the theorem we have to show that $\operatorname{ker}\left(\chi_{y}\right) \subset \mathcal{J}$. This is where we use the structure of the Chern-Hodge ring given by Theorem 10 .

By the proof of [13, theorem 4], there is a basis sequence for $\Omega_{*}^{U} \otimes \mathbb{Q}$ of the following form: $\beta_{1}=\mathbb{C} P^{1}, \beta_{2}=\mathbb{C} P^{2}$, and all $\beta_{i}$ with $i \geq 3$ are holomorphic projective space bundles over an Abelian surface, obtained by projectivizing suitable holomorphic vector bundles. Since the base of these bundles is an Abelian surface, the multiplicativity of the Todd genus shows that the $\beta_{i}$ with $i \geq 3$ are in $\operatorname{ker}\left(\chi_{y}\right)$. Theorem 10 now tells us that we have generators for $\mathcal{C H} \mathcal{H}_{*} \otimes \mathbb{Q}$ all of which except $\beta_{1}=\mathbb{C} P^{1}$ and $\beta_{2}=\mathbb{C} P^{2}$ are in $\operatorname{ker}\left(\chi_{y}\right)$. It is well known that the Hirzebruch genus is surjective onto $\mathbb{Q}\left[\chi_{y}\left(\mathbb{C} P^{1}\right), \chi_{y}\left(\mathbb{C} P^{2}\right)\right]$, compare $[\mathbf{1 4}$, theorem 8 and remark 4]. Therefore, $\operatorname{ker}\left(\chi_{y}\right)$ equals the ideal generated by $E$ and the $\beta_{i}$ with $i \geq 3$. The $\beta_{i}$ with $i \geq 3$ all have nowhere zero holomorphic one-forms obtained by pullback from the base Abelian surface. Of course $E$ has a nowhere zero holomorphic one-form as well, and so we conclude $\operatorname{ker}\left(\chi_{y}\right) \subset \mathcal{J}$.

Remark 12. The inclusions $\mathcal{J} \subset \mathcal{I} \subset \operatorname{ker}\left(\chi_{y}\right)$ hold in $\mathcal{C} \mathcal{H}_{*}$. We only need to tensor with $\mathbb{Q}$ for the proof of $\operatorname{ker}\left(\chi_{y}\right) \subset \mathcal{J}$. The basis sequence from [13] used in that proof is a basis sequence over $\mathbb{Q}$, but not over $\mathbb{Z}$. Whether a suitable basis sequence exists over $\mathbb{Z}$ remains an open problem.

Remark 13. The vanishing of the Hirzebruch genus on Kähler manifolds $X$ with nowhere zero holomorphic one-forms also follows from a result of Green and Lazarsfeld [9, theorem 2]. Their proof, which was the first appearance of the generic vanishing phenomenon in the literature, is much more high-tech than the proof given above. The latter can be summarised by saying that a nowhere zero holomorphic one-form gives a fibration over the circle (Proposition 7), and a fibration over the circle kills the Hirzebruch genus by the elementary mechanism of Lemma 2.

\section{Discussion}

As we mentioned in the introduction, the existence of a nowhere zero holomorphic vector field forces all the Chern numbers to vanish, by a result of Bott [4]. Therefore, the Hirzebruch genus vanishes, giving relations on the Hodge numbers, and one can ask whether there are any further relations satisfied by the Hodge numbers. The answer is no, by the following consequence of the results of [14].

PROPOSITION 14. Let $\mathcal{H}_{*}$ be the Hodge ring of Kähler manifolds, that is the ring of formal $\mathbb{Z}$-linear combinations of Hodge polynomials of compact Kähler manifolds. The kernel of the Hirzebruch genus $\chi_{y}: \mathcal{H}_{*} \longrightarrow \mathbb{Z}[y, z]$ consists precisely of the $\mathbb{Z}$-linear combinations of Hodge polynomials of compact Kähler manifolds admitting nowhere zero holomorphic vector fields.

Proof. By [14, theorem 8], the kernel of $\chi_{y}$ is the principal ideal generated by an elliptic curve. Clearly any product with an elliptic curve admits a nowhere vanishing holomorphic vector field. 
This result should be compared with those of Carrell and Lieberman [6], who proved the vanishing of certain Hodge numbers in the presence of non-trivial holomorphic vector fields with zeros.

Since it is not necessary to tensor with $\mathbb{Q}$, Proposition 14 not only rules out additional $\mathbb{Q}$-linear relations on the Hodge numbers, beyond the vanishing of $\chi_{y}$, but it also rules out congruences.

The results of this paper raise the following problem:

Question 15. Suppose $X$ is a compact Kähler manifold admitting a smooth fibration over the circle. Then does $X$ admit a nowhere zero holomorphic one-form?

This would be the natural converse to Proposition 7 , and one can think of the equality $\mathcal{I}=\mathcal{J}$ in Theorem 11 as evidence in favor of a positive answer.

It is clear that the existence of a smooth fibration over the circle imposes restrictions on the topology of $X$ that go beyond the results on characteristic numbers discussed here. For example, such a fibration gives an exact sequence

$$
1 \longrightarrow K \longrightarrow \pi_{1}(X) \longrightarrow \mathbb{Z} \longrightarrow 1
$$

with a finitely presentable kernel $K$. This gives strong constraints on $\pi_{1}(X)$.

As a consequence of the Enriques-Kodaira classification, Question 15 has a positive answer for surfaces.

PROPOSITION 16. Let $X$ be a compact Kähler surface admitting a smooth fibration over the circle. Then $X$ admits a nowhere zero holomorphic one-form.

Proof. By Lemma 2 the Chern numbers of $X$ vanish. Moreover, if $f: X \longrightarrow S^{1}$ is a smooth fibration, then every continuous map $S^{2} \longrightarrow X$ is homotopic to a map into a fiber of $f$, and so every two-sphere in $X$ has zero selfintersection. In particular, $X$ cannot contain a $(-1)$ curve, and so is minimal. Now by the Enriques-Kodaira classification, see for example [2, p. 188] or [3], $X$ is one of the following:

(i) a surface geometrically ruled over an elliptic curve;

(ii) a hyperelliptic surface;

(iii) a complex torus;

(iv) a minimal elliptic surface of Kodaira dimension one whose only singular fibers are multiple fibers with smooth reduction.

In the first two cases $X$ fibers smoothly and holomorphically over an elliptic curve $E$, and so carries a nowhere zero holomorphic one-form obtained by pullback from $E$. There is nothing to prove for tori, and so (iv) is the only non-trivial case. The topology of the elliptic surfaces arising in (iv) is well understood, and we refer to [8, section 2.7] for the results we use in the following analysis.

For an elliptic fibration $\pi: X \longrightarrow B$ whose only singular fibers are multiple fibers with smooth reduction we mark the points of the base curve $B$ with the multiplicities of the fibers above them and consider $B$ as an orbifold. If this orbifold is bad, then the combination of propositions 7.4 and 7.5 in [8, section 2.7] shows that $X$ is actually as in cases (i) or (iii) above. So in case (iv) we may assume that the orbifold $B$ is good. There is then a finite covering of this orbifold which has no points with multiplicity, and the pullback of $X$ to this covering is a holomorphic fiber bundle with fiber a fixed elliptic curve $E$. The total space of 
this fiber bundle has Kodaira dimension one, and so by the additivity of Kodaira dimension in fiber bundles it follows that the base has Kodaira dimension one. This means that the base has genus $\geq 2$, and so $B$ must be a hyperbolic orbifold.

By lemma 7.3. of [8, section 2.7] we have the following exact sequence

$$
1 \longrightarrow \mathbb{Z}^{2} \longrightarrow \pi_{1}(X) \stackrel{\pi_{*}}{\longrightarrow} \pi_{1}^{\text {orb }}(B) \longrightarrow 1
$$

In particular, $b_{1}(X)=b_{1}(B)=2 g(B)$ or $b_{1}(X)=2+b_{1}(B)=2+2 g(B)$. In the first case $\pi^{*}$ is an isomorphism in degree 1 cohomology, and so every non-trivial homomorphism $\varphi: \pi_{1}(X) \longrightarrow \mathbb{Z}$ factors through $\pi_{1}^{\text {orb }}(B)$. Since $B$ is hyperbolic, this implies that $\varphi$ cannot have finitely generated kernel [10], so in particular $X$ does not fiber smoothly over the circle. In the second case, when $b_{1}(X)=2+b_{1}(B)=2+2 g(B)$, we have an exact sequence

$$
1 \longrightarrow F \longrightarrow A l b(X) \stackrel{\pi_{*}}{\longrightarrow} A l b(B) \longrightarrow 1
$$

such that the Albanese map of $X$ restricts to every elliptic fiber of $X$ as a finite covering map to $F$; compare [3, exercise IX.7 (1)]. We can then take a holomorphic one-form on the Albanese variety $A l b(X)$ which is nowhere zero on $F$. Its pullback under the Albanese map is nowhere zero on $X$. This completes the proof of the Proposition for those elliptic surfaces of Kodaira dimension one that fiber smoothly over the circle.

Acknowledgements. I am very grateful to D. Toledo for useful correspondence, and in particular for reminding me of [1, example 2.1]. This work was done in part at the Institute for Advanced Study in Princeton with the support of The Fund For Math and The Oswald Veblen Fund.

\section{REFERENCES}

[1] J. Amorós, M. Burger, K. Corlette, D. Kotschick and D. Toledo. Fundamental Groups of Compact Kähler Manifolds, Math. Surv. and Monogr., Vol. 44 (Amer. Math. Soc., Providence, R.I. 1996).

[2] W. Barth, C. Peters and A. VAn de Ven. Compact Complex Surfaces (Springer-Verlag, Berlin, 1984).

[3] A. Beauville. Complex Algebraic Surfaces, Second Edition. LMS Student Texts 23 (Cambridge University Press, 1996).

[4] R. BотT. Vector fields and characteristic numbers. Michigan Math. J. 14 (1967), 231-244.

[5] J. B. CARRELL. Holomorphic one forms and characteristic numbers. Topology 13 (1974), 225-228.

[6] J. B. CARRell and D. I. Lieberman. Holomorphic vector fields and Kaehler manifolds. Invent. Math. 21 (1973), 303-309.

[7] R. CREW and D. FRIED. Nonsingular holomorphic flows. Topology 25 (1986), 471-473.

[8] R. Friedman and J. W. Morgan. Smooth four-manifolds and complex surfaces. Ergeb. Math. Grenzgeb. (3), 27., (Springer-Verlag, Berlin, 1994).

[9] M. GReEN and R. LAZARSFELD. Deformation theory, generic vanishing theorems, and some conjectures of Enriques, Catanese and Beauville. Invent. math. 90 (1987), 389-407.

[10] H. B. GRIFFITHS. The fundamental group of a surface, and a theorem of Schreier. Acta Math. 110 (1963), 1-17.

[11] C. D. HACON and S. Kovács. Holomorphic one-forms on varieties of general type. Ann. Sci. Norm. Sup. 38 (2005), 599-607.

[12] D. Kotschick. Characteristic numbers of algebraic varieties. Proc. Natl. Acad. USA. 106, no. 25 (2009), 10114-10115.

[13] D. Kotschick. Topologically invariant Chern numbers of projective varieties. Adv. Math. 229 (2012), 1300-1312.

[14] D. Kotschick and S. SChreIEder. The Hodge ring of Kähler manifolds. Composit. Math. 149 (2013), 637-657. 
[15] W. D. Neumann. Fibering over the circle within a cobordism class. Math. Ann. 192 (1971), 191-192.

[16] M. PopA and C. SCHNELl. Kodaira dimension and zeros of holomorphic one-forms. Ann. of Math. 179 (2014), 1109-1120.

[17] B. SCÁRDUA. Holomorphic Anosov systems, foliations and fibering complex manifolds. Dyn. Syst. 18 (2003), 365-389.

[18] D. TISCHLER. On fibering certain foliated manifolds over $S^{1}$. Topology 9 (1970), 153-154.

[19] Q. ZHANG. Global holomorphic one-forms on projective manifolds with ample canonical bundle. J. Alg. Geometry 6 (1997), 777-787. 
